\title{
Editorial
}

\section{Symmetries, Differential Equations, and Applications: Galois Bicentenary}

\author{
Fazal M. Mahomed, ${ }^{1}$ Asghar Qadir, ${ }^{1,2}$ and Mehmet Pakdemirli ${ }^{3}$ \\ ${ }^{1}$ Differential Equations, Continuum Mechanics and Applications, School of Computational and Applied Mathematics, \\ University of the Witwatersrand, Wits 2050, South Africa \\ ${ }^{2}$ Centre for Advanced Mathematics and Physics, National University of Sciences and Technology, Islamabad, Pakistan \\ ${ }^{3}$ Applied Mathematics and Computation Center, Celal Bayar University, 45040 Manisa, Turkey
}

Correspondence should be addressed to Fazal M. Mahomed; fazal.mahomed@wits.ac.za

Received 3 June 2013; Accepted 3 June 2013

Copyright (C) 2013 Fazal M. Mahomed et al. This is an open access article distributed under the Creative Commons Attribution License, which permits unrestricted use, distribution, and reproduction in any medium, provided the original work is properly cited.

This special issue commemorated the bicentenary of the birth of Évariste Galois who introduced the notion of a group and made fundamental contributions to the solution of polynomial equations. The formal mathematical concept of symmetry thus came from Galois theory. Symmetry was inaugurated and utilized in the solution of differential equations by Sophus Lie in the 1870s. The algebraic approach has thus become of great importance in the theory and applications of differential equations. There was a recent Conference SDEA2012 on this topic held in Johannesburg from 23 to 27 January, 2012. SDEA2012 brought together researchers from all over the world to exchange ideas, share their research findings, and identify future research directions in symmetry and its manifold applications.

We invited the conference participants and researchers in the field to contribute original research articles and reviews to this special issue. We received many contributions which ranged from ordinary to partial differential equations as well as applications. There was one review article on geometric linearization by one of the editors, Asghar Qadir, which covered the state of work in this interesting aspect. The topics of the papers included the following:

(i) Lie theory in approximate and complex differential equations,

(ii) Noether symmetries and their extensions,

(iii) other methods for first integrals and conservation laws, (iv) linearization,

(v) classification schemes,

(vi) invariant characterization,

(vii) stochastic differential equations,

(viii) geometrical methods,

(ix) computational mathematics,

(x) classical mechanics, continuum mechanics, and general relativity,

(xi) equations of finance,

(xii) mathematical modeling in the applied sciences and engineering.

\section{Acknowledgments}

We express our sincere appreciation to the authors for their valuable contributions. Also the crucial work of all the reviewers is greatly acknowledged.

Fazal M. Mahomed Asghar Qadir Mehmet Pakdemirli 


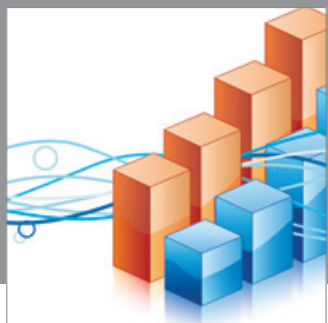

Advances in

Operations Research

mansans

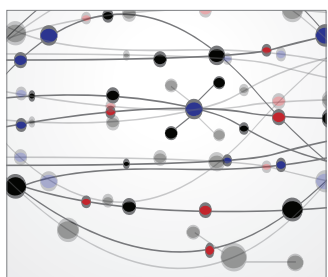

The Scientific World Journal
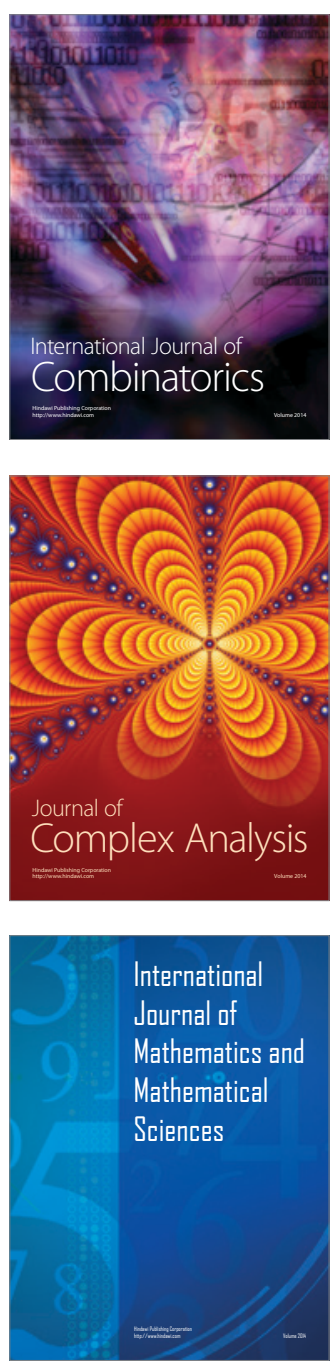
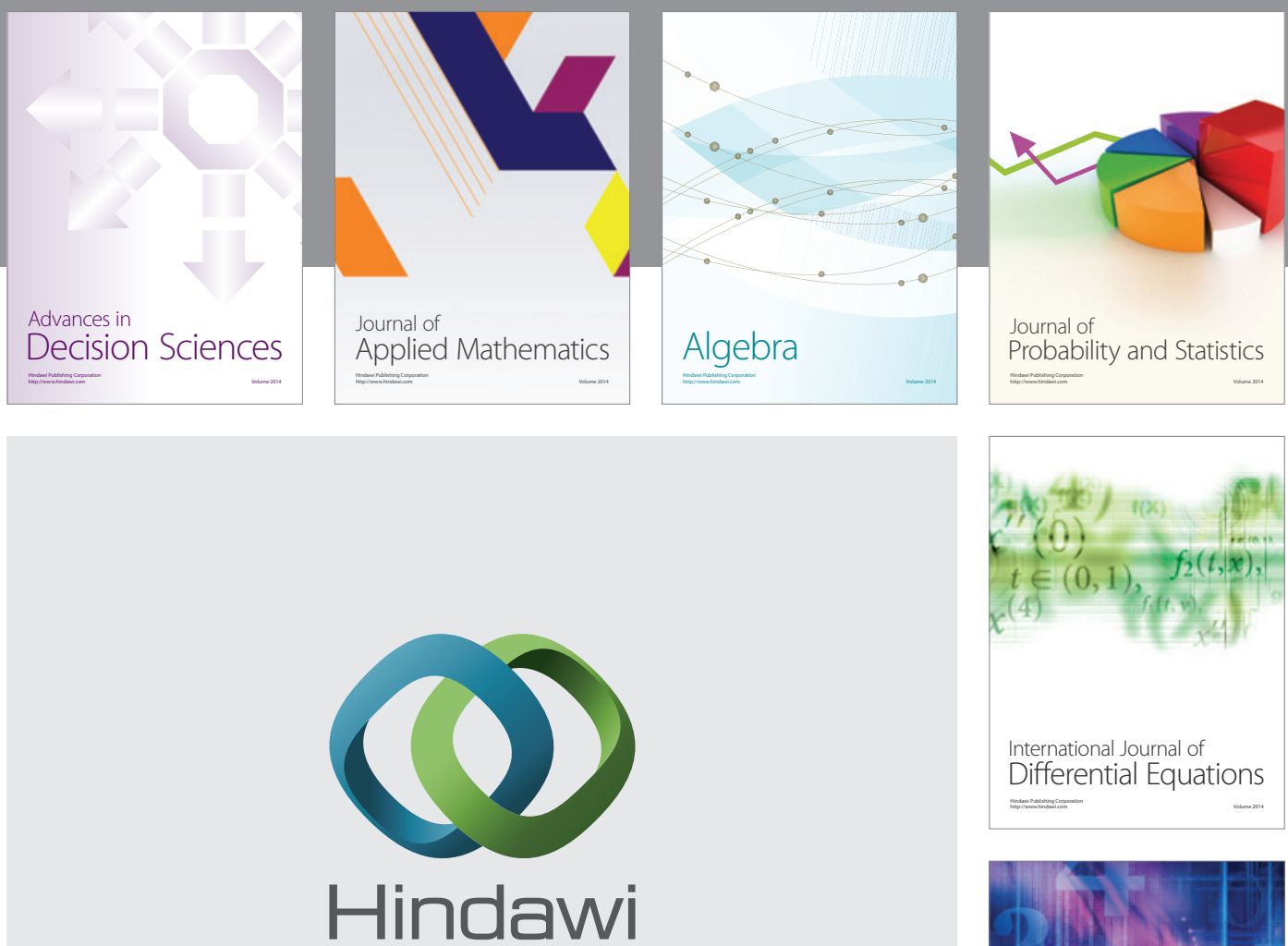

Submit your manuscripts at http://www.hindawi.com
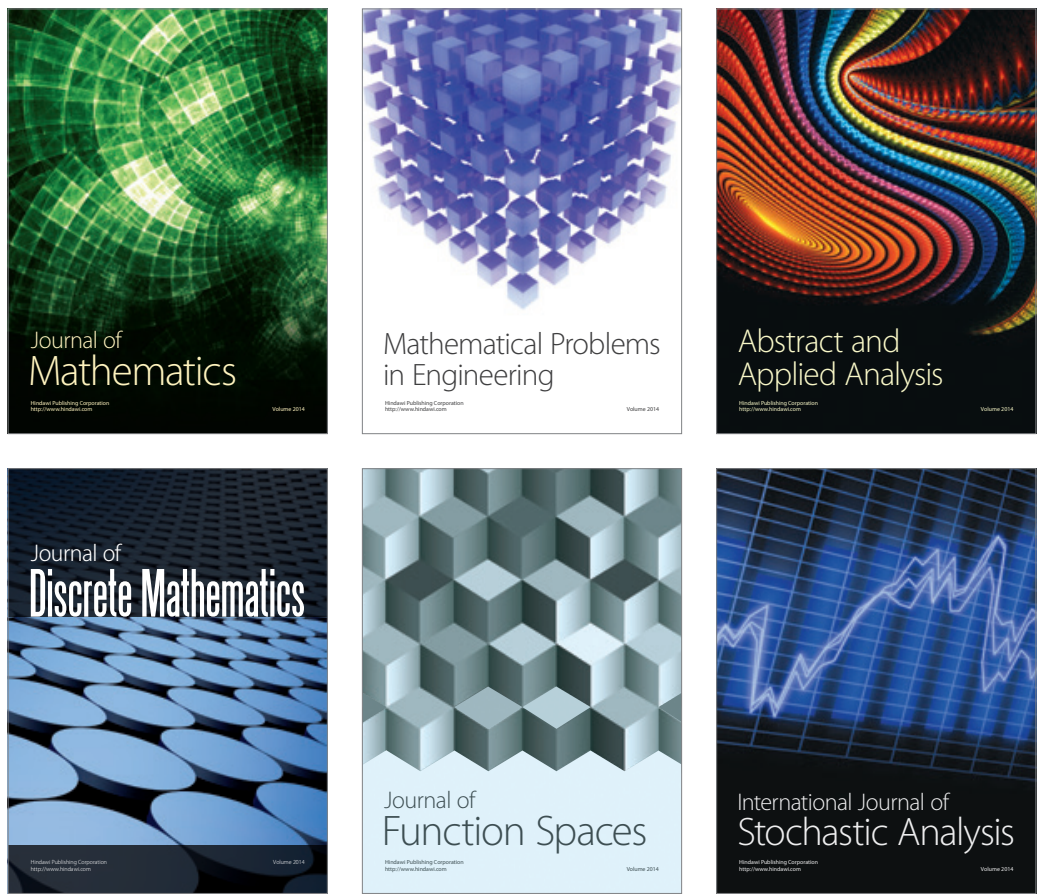

Journal of

Function Spaces

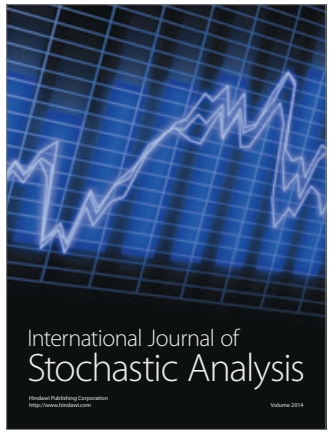

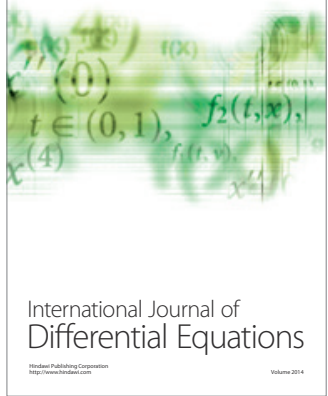
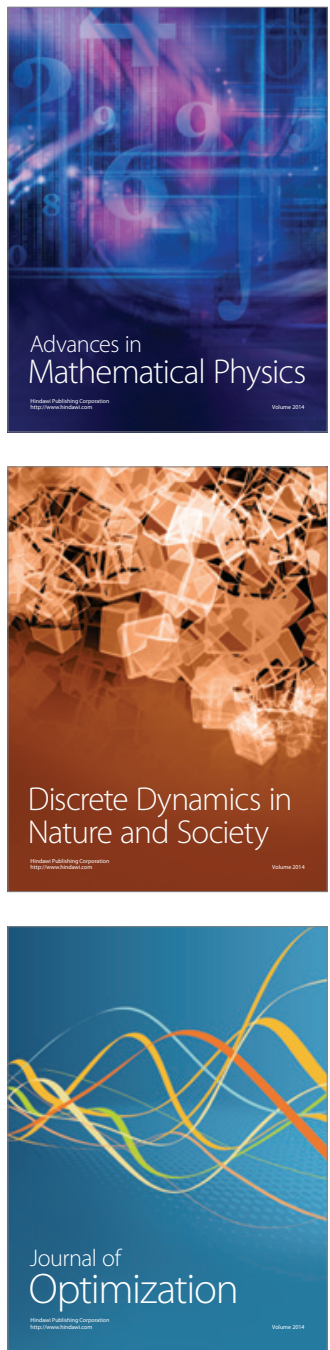